\title{
Elements of Cyber-Socially Physical Systems in "Smart Cities”
}

\author{
BORIS SHUMILOV \\ Department of Applied Mathematics \\ Tomsk State University of Architecture and Building \\ 2 Solyanaya, Tomsk, 634003 \\ RUSSIA \\ sbm05@yandex.ru
}

\begin{abstract}
The implementation of plans to create "smart cities" as one of the most important areas of the digital economy requires the prio rity development of transport infrastructure, ensuring the movement of people and goods within the city and adjacent territories. Safe operation and maximum throughput of the resulting cyberphysical system are possible provi ded that a diagnostic technology is created for transport infrastructure facilities, including video-based road conditions. The author's vision of the problems of mathematical modeling of cyber-physical systems in transport is present ed as a three-level hier archical structure, including environmental sensors at the lower level, data processi ng centers (DPC) at the middle level and a single data storage center for develo ping management decisions at the upper level. Prospects for data center $\mathrm{m}$ odeling based on a m ulti-agent approach and a technical vision algorithm that is proposed to be $\mathrm{i}$ mplemented as a program on a m obile device to identif y objects of tr ansport infrastructure and their defects using stereo metry are explored. The presented algorithm can be used in the planning of road repairs and buildings construction, in the analysis of road and buildings' a ccidents by expert engineers, in the processing of applications of inhabitants' complaints, etc.
\end{abstract}

Key-Words: - Roads as a Cyber-physical systems, Multi-agent method, Photogrammetry

Received: January 3, 2020. Revised: March 10, 2020. Accepted: March 17, 2020. Published: March 31, 2020.

\section{Introduction}

Automation of the diagnostics of sta te transport infrastructure facilities and the relat ed need to develop modules for mathematical modeling, design and production of devices and diagnostic tools as part of the $\mathrm{c}$ ontrol system of the transport $\mathrm{c}$ yberphysical system, which provides physical accessibility of infrastructure and cities, causing an urgent problem within the concept of "smart citi es" as one of the most important directions of digital economy [1]. At first, the quality of the road surface along with the category of the road, its visibility, the width of th e roadway, and the location of the suitable road signs has a significant influence on road safety and traffic flow capacity. Together, they define the concept of "road conditi ons", on which the trajectory and the car's speed depend. According to experts the influence of road cond itions on the occurrence of road traffic accidents, is from $60 \%$ to $80 \%$ of cases [2,3]. Moreover, accidents are one of the most common causes of death in the world. More than $1.35 \mathrm{~m}$ illion people die annually, which is equivalent to the loss by countries affected by this disaster of about $3 \%$ of their gross do mestic product (GDP) [4]. Secondly, according to the results of road accidents' analysis, there is need to identify those who is responsible for the violation of traffic rules (traffic regulations) and the cost esti mate of damaged auto $\mathrm{m}$ otor-vehicle's repair (AMV) [5]. Thirdly, economic and social losse $\mathrm{s}$ due to a decrease in the traffi $\mathrm{c}$ flow's speed and the according road's capacity, caused by the existence of cracks and potholes in the road sur face tend to increase with untimely detection and repair [6].

Quite often controversial situations arise, the investigation and exam ination of which is carried out on available materials, including photo graphs and video $f$ ilming. According to the European Protocol, participants in a road incident can use mobile devices to re cord and register all circumstances (Fig.1).

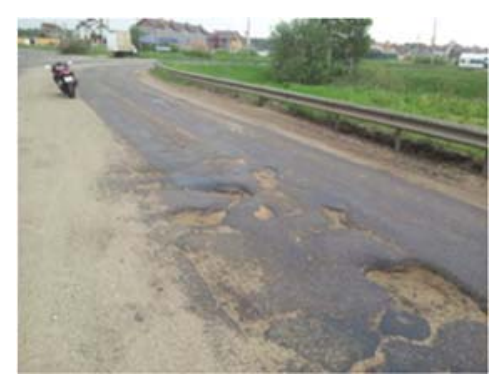

Fig.1. A driver of the motorcycle recorded a general traffic situation

In fig.2, to understand the scale and size of potholes, the driver used a glove as a marker for 
scale [7]. In this situation, the motorcycle owner will have to prove that the road surface does require operating conditions acceptable for road safety.

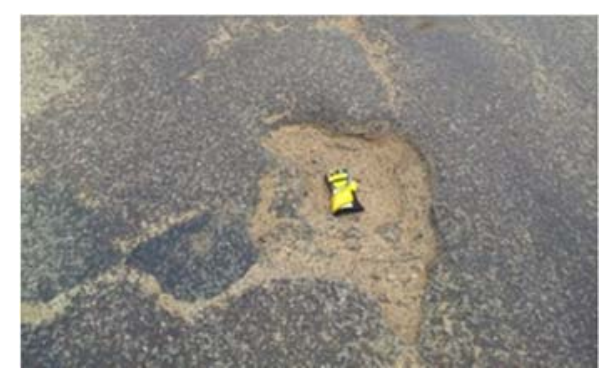

Fig.2. A picture of pothole in the road surface

A definite breakthrough i $\mathrm{n}$ solving the problem of objective diagnostics of road conditions was mobile systems based on laser scanning [8] (Fig.3). However, the high cost of this technology and the lack of information about the texture of road objects complicate the situation.

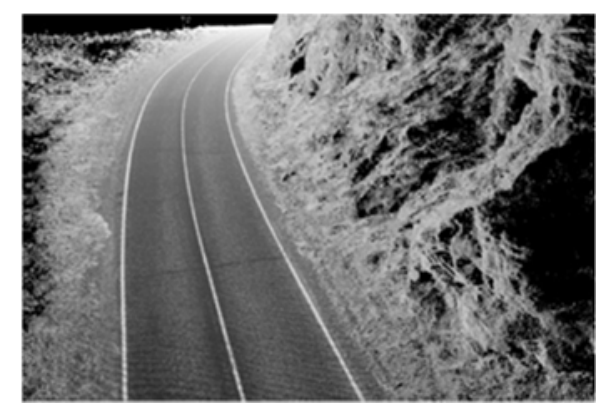

Fig.3. A resulting cloud of laser s canning points includes reflections fro $\mathrm{m}$ people, equipm ent, vegetation, etc. located on the object

The use of modern inexpensive photogrammetric methods [9-15] for determ ining damage and unevenness of the road surface an $d$ structural elements of the road gives a new i mpulse to the development of digital technologies for designing road repairs, increasing $\mathrm{m}$ obility and reducing the cost of work.

Based on the foregoing, the development of autonomous mobile devices capable of "on the spot" to perform the tasks of collecting and analyzing road data, including phot ographs and video, in a form suitable for investigation and exa mination of disputed situations, is an urgent task.

\section{Cyber-physical System of Urban Transport Movement}

The considered above autonomous mobile road data collection and analy sis devices, whic h operate a s part of a single measuring and processing complex, are an affordable example of cyber-physical systems in transport (Fig.4). This also adds a system of stationary city security cameras for road safety and, possibly, a s ystem of unmanned vehicles, which, having a com plex software sy stem of artificial intelligence on board, are able to analy ze independently the surro unding situation, make decisions and learn from previous experience.

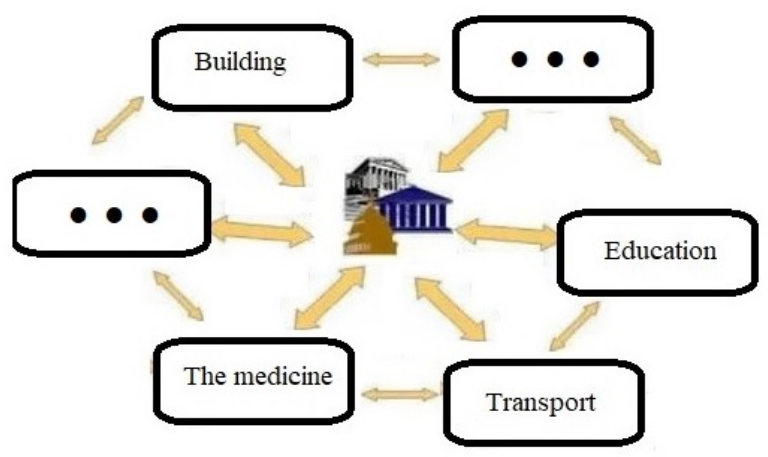

Fig.4. Regional and local authorities

As a result, the totality of such devices form $\mathrm{s}$ a heterogeneous distributed sy stem of artificial intelligence, which shoul d be enriched by some hierarchical structure, which would provide the distribution of tasks between devices, their interaction with the envi ronment and the exchange of information in accordance with fundamental goals and settings (Fig.5).

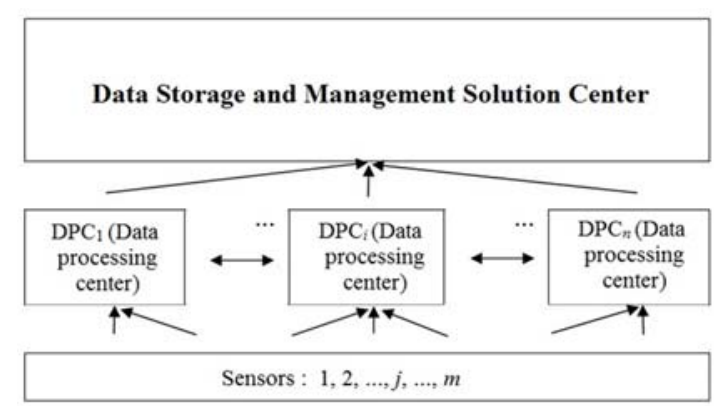

Fig.5. Block diagram of a cyber-ph ysical urban traffic control system

This implies the existence of protocols [16] for their joint work at the lower level of interaction, as well as protocols for sharing in data processing centers (DPC) at the mid-level and further for transfer to a single data center and developm ent of management decisions at the upper level in order to form a global cy ber-socio-physical urban traffic management system as part of the concept of creating "smart cities". The appearance of the word "socio" as applied to the road here is by no $\mathrm{m}$ eans 
accidental, since the road is not a fac tory or field with the prospect of a deserted producti on technology, but an indispensable element of the "smart city", providing accident-free o peration and maximum throughput of the transport system, with the presence in it of active road users, creating the effect of unpredictability of con scious or unconscious behavior of people, drivers, pedestrians and etc.

\subsection{Prospects for Modeling Data Centers Using Programmable Reconfigurable Logic} Arrays

In fig. 6 shows the general organization of a data processing center (DPC) of a cy ber-physical system of urban traffic control at the middle level. Here, PE is a processor element based on a sensor or environmental sensor, a PC is a monitor subsystem based on a personal com puter, UE is a sensor/ sensor interaction control device, TCh is a trunk channel (main bus), $\mathrm{RC}$ is a regular channel (modeling system), DF is a decisiv e field. The modeling system is dashed, in which t he authors of $[17,18]$ use the decisive field on conti nuous VLSI processor matrices.

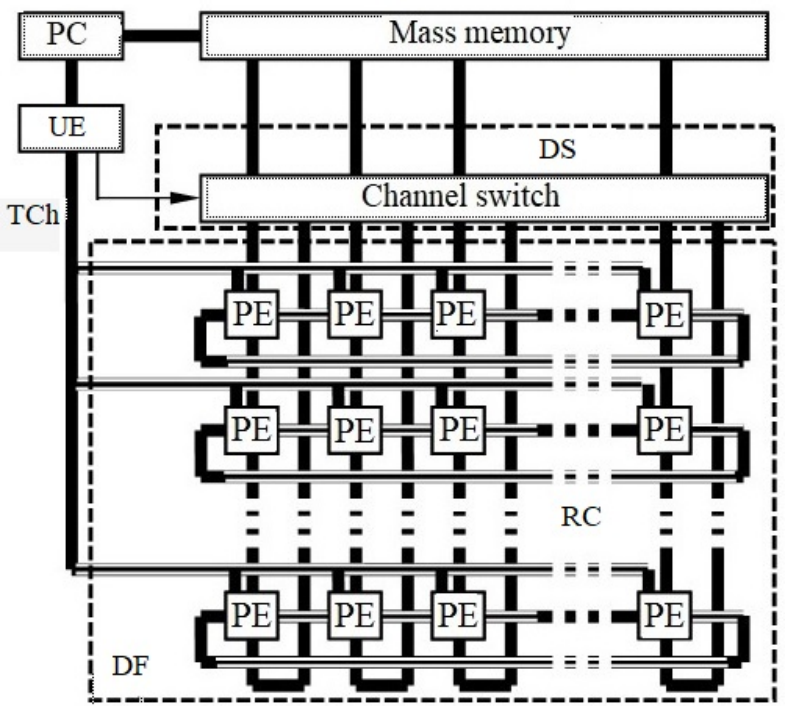

Fig.6. The scheme of organization of the dat a processing center (DPC)

The switching environm ent of each individual sensor or environm ental sensor is shown in fig.7 . Here, the BSG is the matrix adjustment signal generation block; SCU-switching control u nit; TCh-trunk channel.

The undoubted advantage of the hom ogeneous architecture of the processor matrices is its high performance in solving image processing tasks and a huge resou rce of backup and reprogramming for modeling heterogeneous distributed artificial intelligence systems with further equipping with the necessary sensors acco mpanied by programs for primary processing of the received data and transferring control to the following hierarchical processing levels, data storage and management decisions.

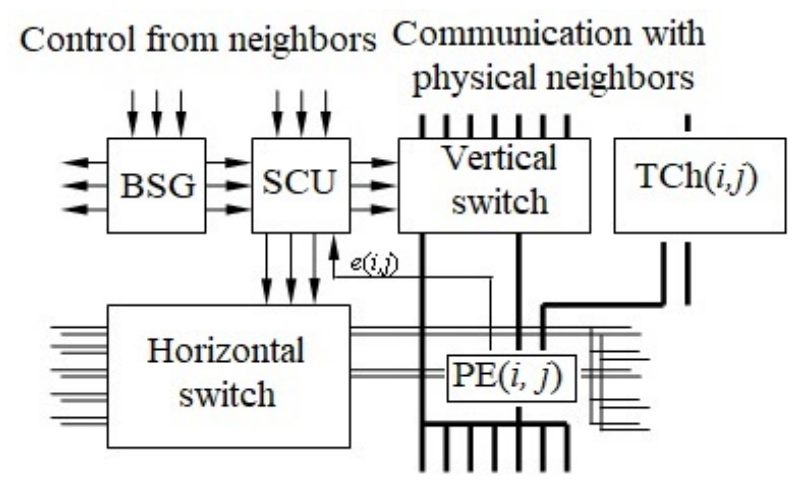

Fig.7. Switching environment of the processor element

\subsubsection{Multi-agent Systems as a Tool for Modeling the Interaction of Environmental Sensors in Transport}

Foundations of multi-agent approach were presented in a series of works [19-22], where also the possibility of applying stochastic models for the design and research of multi-agent systems of economic actors were discussed. For sy stems with swarm intelligence as a model Markov random field was proposed. The agents are locat ed at the top of the final gr aph and are modeled by random variables. In [23-26] was shown the possibility of using multi-agent technology for solving problems of planning in industry and in [27-29] proposed situational approach to the management of resources and developed a m ulti-agent platform for development of intellige nt systems that preserves the scene in the context of the situation, to im prove the quality and efficiency of planning in the process of events' changes, including the development of methods of receiving and processing actual dat a from production resources in real ti me [30]. The study of connected subsets (clusters) of agents deserves special attention. The formation of clusters corresponds to the allocation of road objects, the creation of a production or transport associations. Under the as sumption that agents are located at the vertices of a regular lat tice, an esti mate of the probability of cluster's existence, penetrating the entire system, can s erve the analy tical results, obtained for these lattices in the percolation theory [18]. The critical probabilities of nodes and links 
serve as lower estimation for the agent's coefficients availability, which defines the probabilit $y$ of existence of global cluster and coheren $t$ subsets of agents [31].

\section{Stereo Measurement Solution}

The bibliography [10-14] analyzes the key component of the proposed cy ber-physical system for diagnosing transport infrastructure objects, the role of which is the technical vision system [9], based on the method of photogrammetric processing of stereoscopic images of a three-dimensional 3D object obtained from different angles.

\subsection{Localization and Identification of Projections of Interpolation Points on Images Determined from Reference Frames of a Video Sequence and Forming a Stereo Pair} Highlighting the boundaries of flat images is one of the important auxiliary tasks in recognizing defects in transport infrastructure objects, $\mathrm{i} n$ particular, damage to the road surface. They contain comprehensive information about their form for subsequent analysis [32-35]. As a prelim inary conclusion, we note that the im ages of objects of transport infrastructure a re characterized by the presence of angles formed by the inter-sections of generators (curves or straight lines). $\mathrm{T}$ herefore, for the analysis of such images, it is advisable to use the so-called «angular» filters, in particular, the use of the Harris detector is popular $[33,35]$.

\subsubsection{Experiment Results}

To illustrate the photogrammetric method, let's consider a real stereo pair corresponding to two photographs of a ty pical road cone against a background of damage in the road surface (Fig.8).

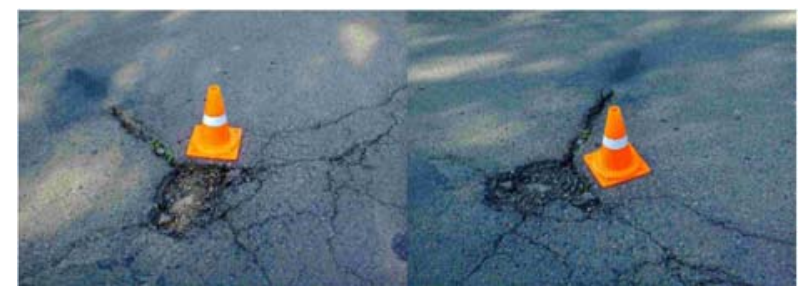

Fig.8. Stereo pair: road cone on the $b$ ackground of damaged road surface

We measured the coordinates of the seven vertices of a three-dimensional object using a ruler. In addition, we fixed the coordinates of the corresponding points on the images in the im age editor with the mouse. Given the coordi nates of the road surface point on the left and right images, one can evaluate the accuracy of the vision algorithm. In our case, the distance from the top of the cone to the asphalt pavement, calcul ated according to the Pythagorean Theorem, was $31.975 \mathrm{~cm}$, which is $0.078 \%$ different from the value of $32 \mathrm{~cm}$ in the technical data sheet.

\subsection{Computer Application}

To test the ability to automatically search for graphic markers and anchor poi nts in photographs we suppose to carry out the implementation of the obtained algorithms on a sm artphone running the Android OS. Re mind that precisely these points must be found on each im age in order $t \quad o$ successfully solve the problem of phot ogrammetry. Due to the peculiarities of the image of the defects of the road way (lack of clear boundaries, the presence of outsiders objects, the insignificance of some defects), it should be possible to " manually" mark the characteristic points on the i mages. The algorithm for creating such an interface that allows selecting the characteristic points by the movement of the graphical cursor is the following. In succession, the left and then the right snapshot of the stereo pair are reset to the smartphone screen (Fig.9).

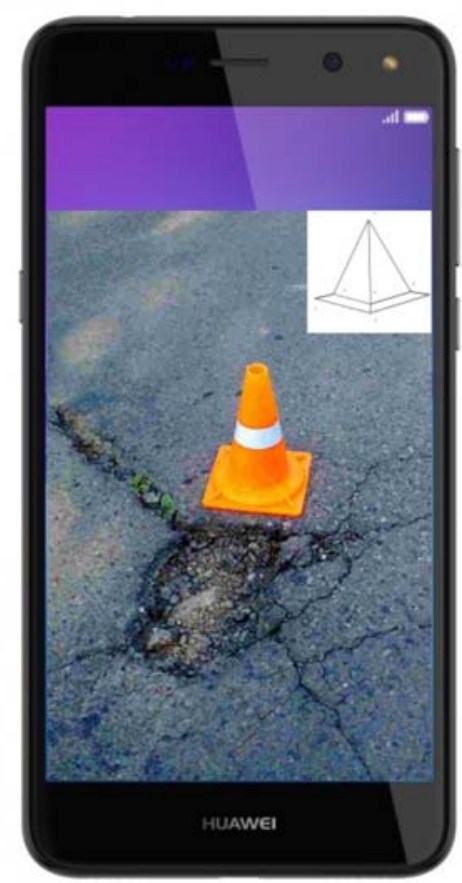

Fig.9. Imitation of wor $\mathrm{k}$ with the $\mathrm{i}$ mage on the smartphone screen

An image of a wire model of a 3D object superimposes the whole i mage. On the model, the 
characteristic points of the object flash, and the user by the movement of the graphic cursor selects th $\mathrm{e}$ corresponding point in the photo. After processing both images of the stereo pair, the algorithm forms linear perspective transformation matrices in the smartphone's memory (a so-called calibration). Then by a command sent the user select $s$ an arbitrary point in each sna pshot of the stereo pair. It is proposed to choose from the appropriate menu the test mode (if it is a characteristic point belongin $g$ to the object), or the test mode for the probing of the Pythagorean Theorem (if it is a point on the road base), or to c ontinue to work. Next, by a co mmand sent the user sel ects the second poi nt in each snapshot of the stereo pair; and the algorithm calculates the distance between selected points (another test). Next, the us er selects the third and so on points in each picture of the stere o pair when prompted. As the array of characteristic points filled, it bec omes possible using the $t$ riangulation technology to constr uct a three-dim ensional mathematical model of a road surface defect. Then, based on the obtained model, the area and volume of the geometric figure that characterizes this particular damage to the road surface are calculated. A further task is to prepare an order for the repair work to eliminate this road defe ct. The full cy cle of work s on the recognition of road surface damage is shown in fig. 10 .

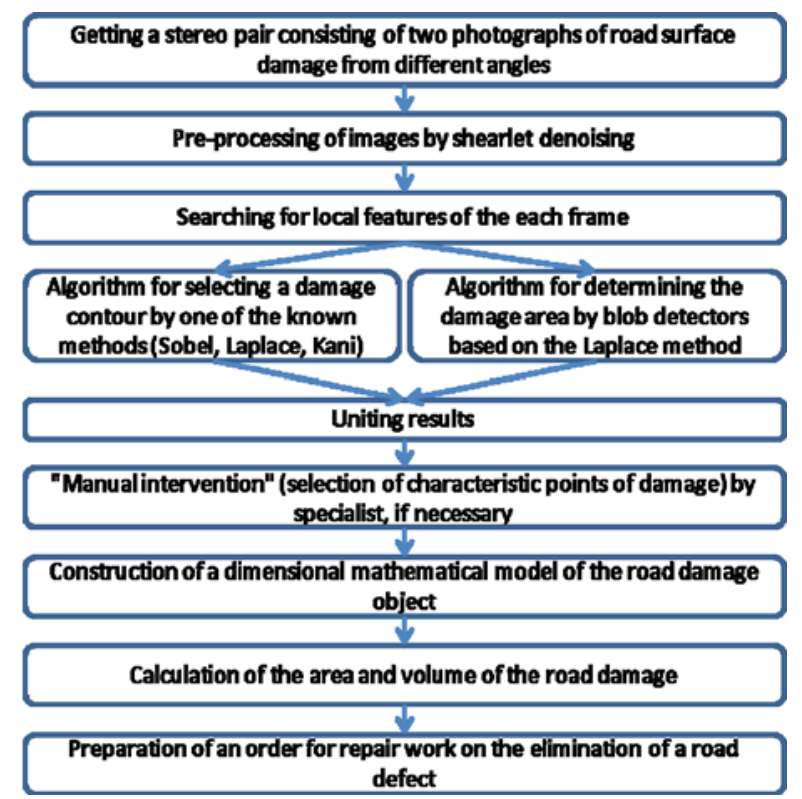

Fig.10. The structure of the software-algorithmic complex recognition of road surface damage

\section{Conclusion}

The practical application of the algorithm stated in the report is quite wide, including whe $\mathrm{n}$ fixing and determining the actual dimensions of dam age to buildings' construction based on the mea sured values of body surface points on the left and right images (Fig.11).

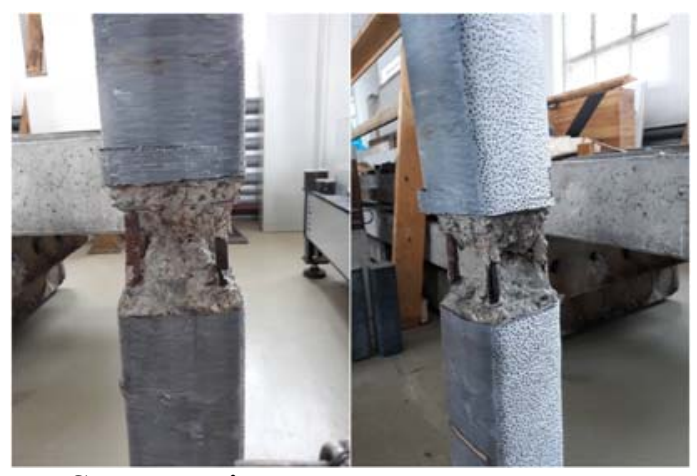

Fig.11. Stereo pair: two photos of a concrete bulk damaged in a building accident

Examination of the na ture and the list of damages to the $\mathrm{b}$ uildings' construction (Fi g.12) provides for a detailed fixation of the dam ages to determine the possibilit $\mathrm{y}$ of their $\mathrm{f}$ ormation and involvement in the event under investigation. The corresponding act records the results of the visual inspection of the damaged buil dings' construction and photographing. Photographs and inspection report for bu ildings' construction are a mandatory annex to the expert op inion or re port on the assessment of the cost of repairing the buildings' construction.

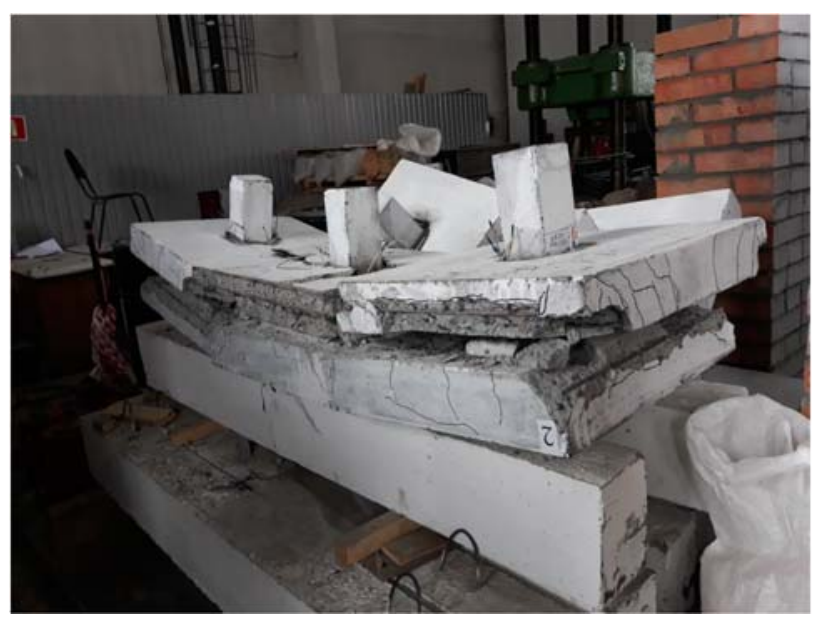

Fig.12. Ruins of the collapsed concrete floor, submitted for examination

Of course, the photogrammetric method is a weighty argument in solving controversial situations, and the determination of the actual size of damage to the elements of the buildi ngs' construction from photographs and video footage is an urgent task. In conclusion, we should say that the remote collection of data on b uildings' construction defects could be 
much more efficient using the methods described in this report.

\section{References:}

[1] V. Kupriyanovsky, D. Namiot, S. Si nyagov, Cyber-physical systems as a base for digital economy. International Journal of Open Information Technologies, Vol.4, No.2, 2016. http://injoit.org/index.php/j1/article/view/266/2 11

[2] A.A. Urfi, S. Khalil, M.F. Hoda, Risk factors for road traffic accident $\mathrm{s}$ with head i njury in Aligarh. International Journal of Medical Science and Public Health, Vol.5, 20 16, pp. 2103-2107.

[3] T. Abdi, B. Hailu, A.T. Andualem, P.H.A.J.M. Gelder, M. Hagenzieker, C.-C. Carbo n, Road Crashes in Addis Ababa, Ethiopia: Em pirical Findings between the Years 2010 and 2014 . AFRREV, Vol.11, 2017, pp. 1-13.

[4] Global status report on road safety 2018. http://www.who.int/violence\_injury\_preventi on/road\_safety\_status/2018/en/

[5] A. Morales, L.J. Sa nchez-Aparicio, D. Gonzalez-Aguilera, M.A. Gutierre z, A.I. Lopez, D. Hernandez-L opez, P. RodriguezGonzalvez, A new approach to energy calculation of road acc idents against fixed small section elements based on close-range photogrammetry. Remote sensing, Vol.9, No.1219, 2017, pp. 1-18.

[6] G. Bao, Road distress analysis using 2D and $3 D$ information, The University of Toledo Digital Repository Theses and Disser tations, 2010.

[7] E.A. Esharov, A.V. Davydov, V.S. Kalinichenko, The use of data processing algorithms for mobile video measurements for fixing and studying damage to motor vehicles. Perspective Information Technologies (PIT 2018) Proceedings of the International Scientific and Technical Conference. 2018, pp. 913-916.

[8] B.M. Shumilov, A.N. Baigulov, A stud y on modeling of road pavements based on laser scanned data and a novel type of approximating hermite wavelets. WSEAS Transactions on Signal Processing, Vol.11, 2015, pp. 150-156.

[9] P. Elugachev, B. Shum ilov, Development of the technical vision algorithm. MATEC Web of Conferences, Vol.216, 04003 / In: Polytransport Systems-2018, 2018.
[10] D.F. Rogers, J.A. Ad ams, Mathematical elements for computer graphics. McGraw-Hill, 1990.

[11] R. Tsai, A versatile camera calibration technique for high-accuracy 3D machine vision metrology using off-the-shelf TV cameras and lenses. IEEE J. of Robotics and Automation, Vol.3, 1987, pp. 323-344.

[12] C. Slama (ed.), Manual of Photogrammetry. American Society of Photogrammetry, 1980.

[13] A.J. Davison, I.D. Reid, N.D. Molton, O. Stasse, MonoSLAM: Real-Time Single Camera SLAM. IEEE Trans. Pattern Anal. Machine Intell., Vol.29, 2007, pp. 1052-1067.

[14] A. Chiuso, P. Favaro, H. Jin, S. Soatto, Structure from motion causally integrated over time. IEEE Trans. Pattern Anal. Machine Intell., Vol.24, 2002, pp. 523-535.

[15] R. Hartley, A. Zisser man, Multiple View Geometry in Computer Vision. 2nd edn. Cambridge University Press, 2004.

[16] V.Ya. Tsvetkov, Control with the use of cyberphysical systems. Perspectives of Science \& Education, Vol.3, No.2 7, 2017, pp. 55-60. http://psejournal.wordpress.com/archive17/17$03 /$

[17] V.A. Vorob'ev, N.L. Ere mina, N.V. Lakhodynova, Analysis of algorithms of the structure reconstruction of a processor matrix, Avtometriya, No.3, 19 96, pp. 69-77. (in Russian)

[18] N.V. Lakhodynova, On percolation threshold in homogeneous structures. Avtometriya, No.3, 2003, pp. 35-42. (in Russian)

[19] S.N. Chapman, The Fundamentals of Production Planning and Control. Prentice Hall, 2006.

[20] M. Wooldridge, An Introduction to Multi-Agent Systems. Wiley, 2009.

[21] D. Easley, J. Kleinberg, Networks, Crowds, and Markets: Reasoning About a Highly Connected World. Cambridge University Press, 2010.

[22] N.R. Jennings, M.J. Wooldridge (eds.), Agent Technology: Foundations, Applications, and Markets. Springer, 2012.

[23] V.I. Gorodetsky, P.O. Skobelev, Industrial applications of multi-agent technology: reality and perspectives. SPIIRAS Proc., Vol.55, No.6, 2017, pp. 11-45.

[24] V.A. Vittikh, T.V. Moise eva, P.O. Skobelev, Making decisions on the basis of consensus using multi-agent technologies. Ontol. Des., Vol.2, No.8, 2013, pp. 20-25. (in Russian)

[25] P. Skobelev, Towards autonomous AI systems for resource management: applications $i \quad n$ 
industry and lessons lear ned. Proceedings of the XVI International Conference on Practical Applications of Agents and Multi-Agent Systems (PAAMS 2018). LNAI, Vol.10978, 2018, pp. 12-25.

https://doi.org/10.1007/978-3-319-94580-4_2

[26] G. Rzevski, P. Skobelev, Complexity. Wit Press, 2014.

[27] P. Skobelev, et al., Practical approach and multi-agent platform for designing $r$ eal time adaptive scheduling systems. Proceedings of the XII International Conference on Practical Applications of Agents and Multi-Agent Systems (PAAMS 2014). CCIS, Vol.0430, 2014, pp. 1-12.

[28] P. Skobelev, Multi-agent systems for real time adaptive resource management. In: Leitão, P., Karnouskos, S. (eds.) Industrial Agents: Emerging Applications of Software Agents in Industry, 2015, pp. 207-230.

[29] J. Leung, Handbook of Scheduling: Algorithms, Models and Performance Analysis. Chapman \& Hall, 2004.
[30] I. Mayorov, P.S kobelev, Towards thermodynamics of real time scheduling. Int. J. Des. Nat. Ecodynamics, Vol.10, No. 3, 2015, pp. 213-223.

https://doi.org/10.2495/dne-v10-n3-213-223/

[31] A. Lada, S. Smirnov, The Multi-agent Method for Real Time Production Resource-Scheduling Problem in Recent Research in Control Engineering and Decision Making. Studies in Systems, Decision and Control/ICIT-2019, 2019, p. 103-111.

[32] M. Sonka, V. Hlavac ， R, Boy le, Image Processing. Analysis and Machine Vision. Thomson, 2008.

[33] Harris affine regiond etector, https://en.wikipedia.org/wiki/Harris_affine_reg ion detector

[34] T. Tuytelaars, K. Mikolajczyk, Local Invariant Feature Detectors: A Survey. Foundations and Trends in Computer Graphics and Vision, Vol.3, No.4, 2007, pp. 177-280.

[35] C. Harris, M. Stephens, A combined corner and edge detector. Fourth Alvey Vision Conference, 1988, pp. 147-151.

[36] Atef Ibrahim, Hamed Elsimary, Abdullah Aljumah, Fayez Gebali, A New Processor Array Structure for Protein Sequence Alignment using Sm ith-Waterman Algorithm, WSEAS Transactions on Circuits and Sy stems pp. 282-293, Volume 14, 2015

[37] Atef Ibrahim, Hamed Elsimary, Abdullah Aljumah, Fayez Gebali, New Processor Array Architecture for DNA Sequence Alignment Using Stevens-Song Algorithm, WSEAS Transactions on Circuits and Systems, pp. 320330, Volume 14, 2015 